\title{
Preparation of superparamagnetic iron oxide nanoparticles and investigation of their interaction with cells
}

\author{
Wid Mekseriwattana ${ }^{\mathrm{a}}$, Supreeya Srisuk ${ }^{\mathrm{b}}$, Yotsakorn Tantiapibalkun ${ }^{\mathrm{b}}$, Kanlaya Prapainop ${ }^{\mathrm{a}, \mathrm{b}, *}$ \\ a School of Materials Science and Innovation, Faculty of Science, Mahidol University, \\ Bangkok 10400 Thailand \\ b Department of Biochemistry, Faculty of Science, Mahidol University, Bangkok 10400 Thailand
}

*Corresponding author, e-mail: kanlaya.pra@mahidol.edu

Received 19 Jul 2018

Accepted 24 Feb 2019

\begin{abstract}
Superparamagnetic iron oxide nanoparticles (SPIONs) have been widely studied in biomedical applications such as bioimaging through magnetic resonance imaging and drug delivery. The successful uses of SPIONs depend on nanoparticles stability in biological environment and their interactions with cells. Hence these two factors are crucial for improvement of nanoparticle design in these applications. In this work, SPIONs were synthesized with silica (SSPIONs) and amine (A-SPIONs) surface modifications providing hydroxyl and amine functionalities, respectively. The colloidal stabilities of SPIONs were evaluated as hydrodynamic size in different biological relevance media. The results showed that bare SPIONs were unstable and highly aggregated when exposed to cell culture media. Coating with silica and amine could effectively stabilized the nanoparticles as evidenced by reduction of hydrodynamic diameters. In addition to surface modification, supplementation of serum proteins to cell culture media also reduced the aggregations. Furthermore, both S-SPIONs and A-SPIONs showed no cytotoxicity effect on human breast cancer cells (MCF-7) with cell viability remained over $80 \%$. Hence this study showed the role of surface modification of bare SPIONs with silica and amine functionalization and serum supplement to stabilize nanoparticle stability in biological environment. These two surface coating SPIONs were not only non-toxic to the cells, but also have surface functionalities that could be further conjugated with desired biomolecules for more specific targeting especially in cancer targeting for diagnosis or therapeutic applications.
\end{abstract}

KEYWORDS: superparamagnetic iron oxide nanoparticle, biological media, cytotoxicity

\section{INTRODUCTION}

Superparamagnetic iron oxide nanoparticles (SPIONs) are inorganic nanoparticles which exhibit intrinsic magnetic properties when exposed to external magnetic field. SPIONs have been explored and utilized in various biomedical applications such as bioimaging $^{1}$, biological separation ${ }^{2,3}$, drug delivery and cancer therapy ${ }^{4-6}$. Several methods have been developed for SPIONs synthesis in aqueous solvent ${ }^{7,8}$. However, in order to acquire an effective use of SPIONs in biological applications, the particles are required to be stable when exposed to biological systems. In contrast to aqueous media, biological systems contain various extra components such as metal cations and anions, proteins, amino acids and other biomolecules. It has been shown that these components could alter the surface properties of nanoparticles (NPs) resulting in different NPs behaviour and different NPs-cell interactions.
Aqueous-stable NPs tend to form aggregates in physiological systems such as serum or cell culture media $^{9}$. Here, surface coatings play important roles in maintaining NPs stability. Biopolymers such as chitosan and dextran are commonly used for SPIONs coatings, providing stabilizing effect and prevent binding of the NPs with environmental proteins ${ }^{4,10}$. $\mathrm{pH}$ responsive polymers like poly(methacrylic acid) are known for its ability to maintain NPs size in response to physiological $\mathrm{pH}^{11}$. Silica coatings are also widely used as protective shells for NPs providing stabilized surface with various functional groups for further bioconjugation ${ }^{12}$.

Apart from intrinsic surface properties of the NPs, serum protein is another key factor in NPs stability. Many studies have been conducted and shown that NPs can interact with proteins in their environment forming protein coronas ${ }^{13,14}$. The protein-NPs complex could eventually result in ei- 
ther enhancing or worsen their stability. In this work, aiming to acquire various functionalized surface, silica was chosen as a coating for SPIONs providing hydroxyl and amine functionalization. The stability of the synthesized NPs in cell culture media was characterized in terms of hydrodynamic diameters and zeta potential to further clarify the effect of the coating materials on colloidal stability along with their interactions with serum proteins in the media. Cellular toxicity of MCF-7 cells in response to the surface modifications of SPIONs in different media were investigated to elaborate the effect of colloidal stability and environmental proteins to NPs and cells interaction.

\section{MATERIALS AND METHODS}

\section{Materials}

$\mathrm{FeCl}_{2} \cdot 4 \mathrm{H}_{2} \mathrm{O}, \quad \mathrm{FeCl}_{3} \cdot 6 \mathrm{H}_{2} \mathrm{O}$, ammonia solution (28-30\%), tetraethyl orthosilicate (TEOS), 3-aminopropyl triethoxysilane (APTES), N,Ndimethyl formamide (DMF) and toluene were obtained from Sigma-Aldrich. 3-(4,5-Dimethyl-2thiazolyl)-2,5-diphenyl-2H-tetrazolium bromide (MTT) was obtained from PanReac AppliChem. Dulbecco's Modified Eagle's Medium (DMEM), fetal bovine serum (FBS) and Penicillin/Streptomycin $(\mathrm{P} / \mathrm{S})$ were obtained from Gibco. Dimethyl sulphoxide (DMSO) was obtained from Fisher Scientific. All of the chemicals were reagent grade and used without further purification.

\section{Synthesis of SPIONs}

The core SPIONs and further functionalization were carried on following a method reported by Jang and $\mathrm{Lim}^{15}$. The synthetic scheme is shown in Fig. 1. For the core SPIONs, $\mathrm{FeCl}_{3} \cdot 6 \mathrm{H}_{2} \mathrm{O}$ and $\mathrm{FeCl}_{2} \cdot 4 \mathrm{H}_{2} \mathrm{O}$ were mixed with stoichiometric ratio of $2: 1$ under $\mathrm{N}_{2}$ atmosphere. The solution was heated to $80^{\circ} \mathrm{C}$ then ammonium hydroxide was added. Black precipitates were formed immediately and the reaction was prolonged for $20 \mathrm{~min}$. The resulted SPIONs were collected by magnetic decantation and stored as stock solution.

\section{Synthesis of silica-coated SPIONs (S-SPIONs)}

Coating of SPIONs with silica was performed following previously reported method ${ }^{15}$. Briefly, SPIONs were dispersed in $20 \%$ ethanol and sonicated for $15 \mathrm{~min}$. The solution $\mathrm{pH}$ was adjusted to 9 using ammonium hydroxide then TEOS was added under continuous sonication. The mixed solution was stirred for $4 \mathrm{~h}$. The precipitates were washed three times with deionized water and collected by centrifugation (15000 rpm).

\section{Synthesis of amine-functionalized SPIONs (A-SPIONs)}

S-SPIONs were dispersed in water to the concentration of $1 \mathrm{mg} / \mathrm{ml}$. Solution of APTES in ethanol (10\%v, $10 \mathrm{ml}$ ) was added drop-wise to the solution. The reaction mixture was stirred at room temperature for $1 \mathrm{~h}$ then the temperature was raised to $90^{\circ} \mathrm{C}$ and stirred for another $1 \mathrm{~h}$. The obtained A-SPIONs were washed with water and collected by magnetic decantation.

\section{Nanoparticle characterization}

Functional groups and surface coatings of the particles were studied by infrared spectrometry using attenuated total reflection mode (Perkin Elmer Frontier FTIR spectrometer and Bruker ALPHA FTIR spectrometer).

\section{Colloidal stability evaluation of nanoparticles}

Colloidal stability of the nanoparticles was determined by measurement of hydrodynamic diameter and zeta potential using dynamic light scattering (DLS) technique with Malvern Zetasizer ZS. The SPIONs samples were dispersed in water, DMEM, DMEM with $1 \%$ FBS and DMEM with $10 \%$ FBS at final concentration of $0.03 \mathrm{mg} / \mathrm{ml}$.

\section{Cell cytotoxicity assay}

MCF-7 human breast cancer cells were cultured in DMEM medium supplemented with 10\% FBS and $1 \% \mathrm{P} / \mathrm{S}$. Cells were kept at $37^{\circ} \mathrm{C}$ under $5 \% \mathrm{CO}_{2}$ in a humidified incubator. Cells were seeded onto a 96well plate at cell density of $8 \times 10^{3}$ cells/well and incubated. After $24 \mathrm{~h}$, the media was replaced with freshly prepared media containing S-SPIONs and ASPIONs with different concentration of $0.05,0.1$, and $0.4 \mathrm{mg} / \mathrm{ml}$ and incubation time was varied to 6 and $24 \mathrm{~h}$. After incubation, cells were washed with PBS buffer 3 times and followed by addition of MTT solution. Upon 3-h incubation, the media was removed and DMSO was added to each well. Cell viability was determined by measuring absorbance at $540 \mathrm{~nm}$ using a microplate reader (Tecan Spark $10 \mathrm{M})$.

\section{RESULTS AND DISCUSSION}

The SPIONs were synthesized according to the scheme in Fig. 1. The functionalization of SPIONs was characterized by FTIR spectroscopy and 


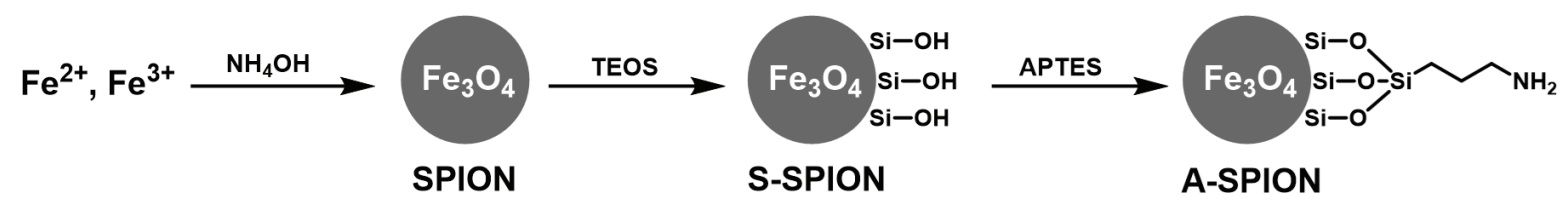

Fig. 1 Synthetic scheme of SPION, S-SPION, and A-SPION.

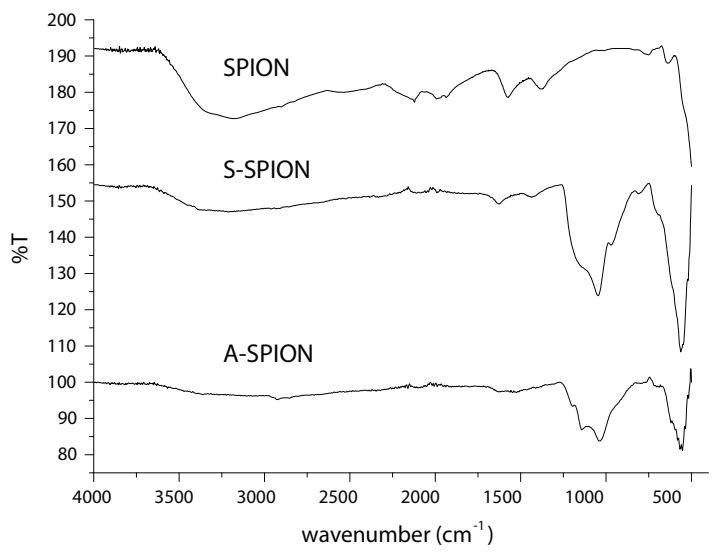

Fig. 2 FTIR spectra of SPION, S-SPION, and A-SPION.

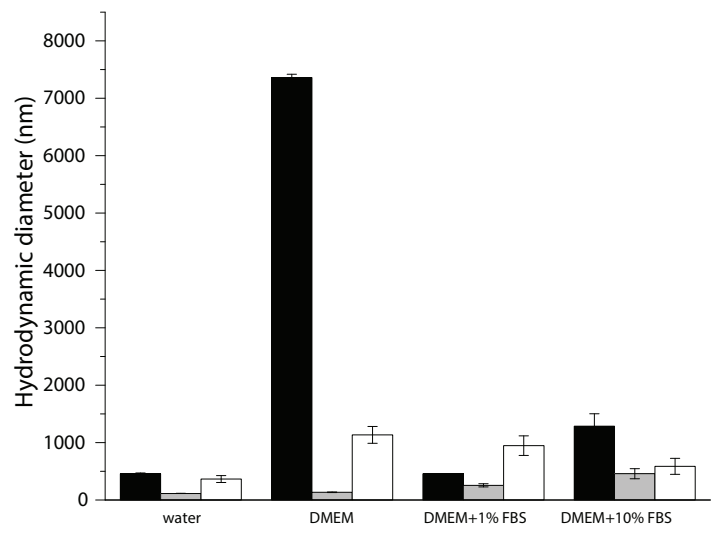

Fig. 3 Hydrodynamic diameters of SPIONs with different coatings in biological relevant media. Black, grey and white bars represent SPIONs, S-SPIONs, and A-SPIONs, respectively.

is shown in Fig. 2. Uncoated SPIONs showed characteristic peak of iron oxide at around $500 \mathrm{~cm}^{-1}$. Coating of silica in S-SPIONs and A-SPIONs could be confirmed by strong peaks at $1100 \mathrm{~cm}^{-1}$ corresponding to Si-O bonds. For A-SPIONs, FTIR peaks of N-H bonds could not be clearly observed, however, the functionalization could be confirmed by zeta-potential measurement. S-SPIONs have zeta-potential value of $-31.5 \mathrm{mV}$ which originated from the hydroxyl groups. After functionalization with APTES, A-SPIONs showed positive zetapotential value of $19.8 \mathrm{mV}$ which indicates amine groups. Hydrodynamic diameters of each sample in different biological relevant media are shown in Fig. 3 Bare SPIONs showed sizes around $450 \mathrm{~nm}$ in water. Coating of SPIONs with silica resulted in hydroxyl functionalized surface which showed stabilizing property evidenced from the reduction of hydrodynamic diameters to $100 \mathrm{~nm}$. In contrast, further reaction of S-SPIONs with APTES to yield amine groups resulted in aggregation of the NPs with size $365 \mathrm{~nm}$ which could be induced by positively charged amine functional groups presenting at the surface.

Upon exposure to cell culture media, bare SPIONs and A-SPIONs strongly formed large aggregates in the range of $\mu \mathrm{m}$. This could be due to the presence of complex ions presented in the media which interact with the SPIONs through charges and destabilized the surfaces ${ }^{9}$. Surprisingly, silica coatings on S-SPIONs showed promising stabilizing effect to the NPs with the maintained size $130 \mathrm{~nm}$. Addition of serum proteins to final concentration of $1 \%$ greatly reduced the hydrodynamic diameters of all NP samples. Serum proteins could interact to the SPIONs surface, thus forming proteins coronas and stabilized the NPs. However, too high concentration of the proteins could in turn result in aggregation of the NPs as observed from the increase of hydrodynamic diameters of bare SPIONs and SSPIONs in media containing $10 \%$ FBS. In contrast, hydrodynamic diameters of A-SPIONs surprisingly reduced to $600 \mathrm{~nm}$ with high FBS concentration. These results elaborated that SPIONs with different surface properties could react differently to environmental proteins and should be further studied in the aspect of protein characterization to fully understand the phenomena.

To investigate the effect of stabilization on cellular interaction, cytotoxicity of the SPIONs on MCF7 cells was investigated (Fig. 4). The cells were incubated with S-SPIONs and A-SPIONs at various concentrations and cell viabilities were evaluated 

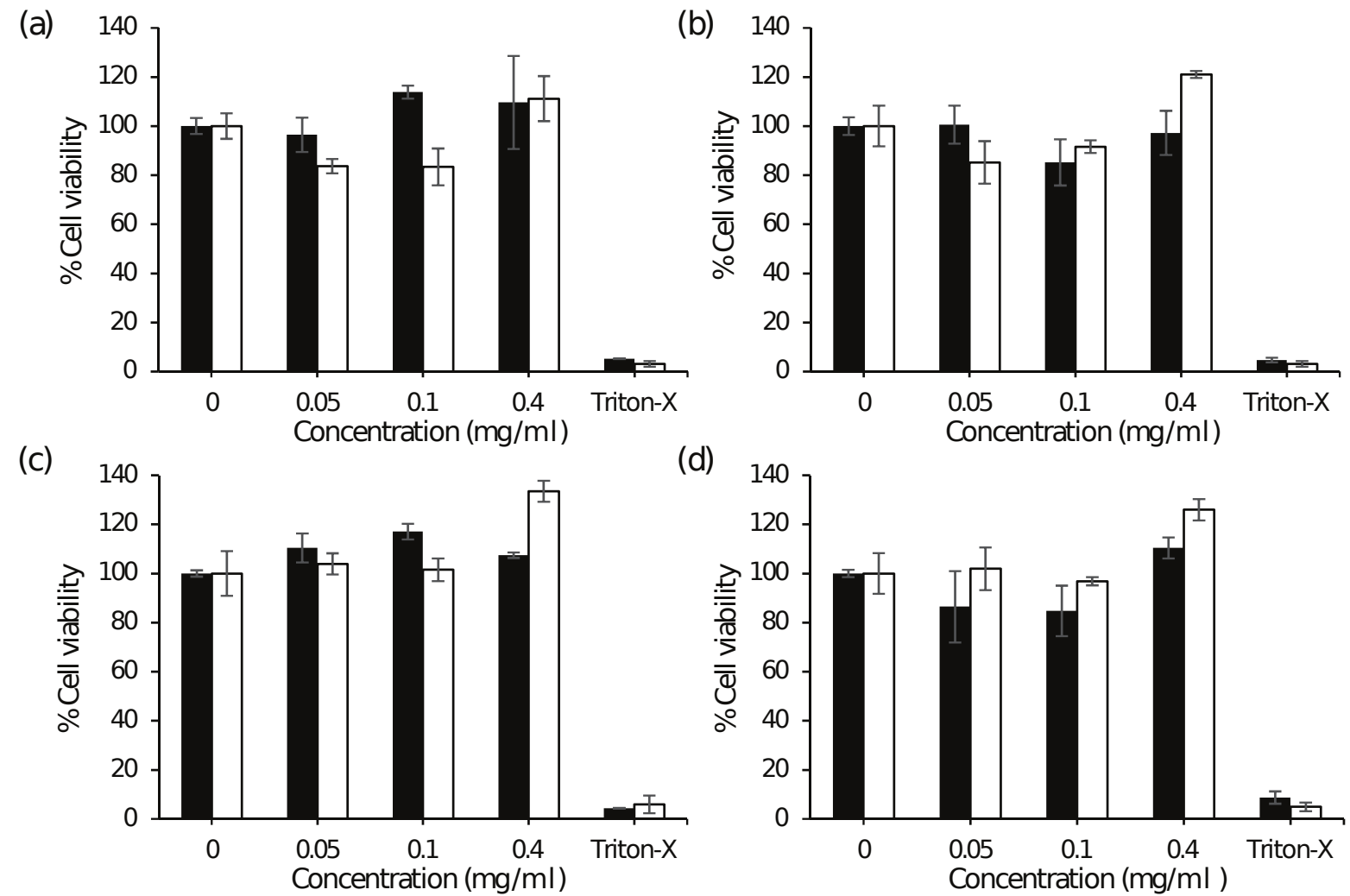

(d)

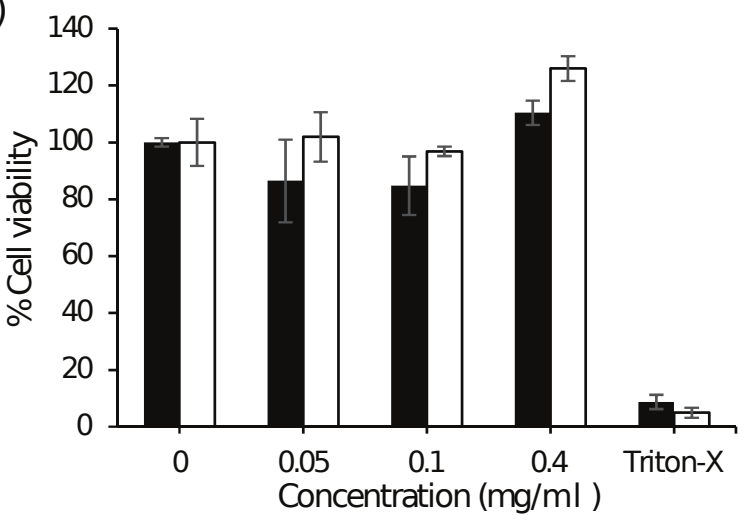

Fig. 4 Cell viability of MCF-7 cells after incubating with NPs in (a,b) DMEM+1\% FBS for (a) 6 and (b) 24 h, (c,d) DMEM+10\% FBS for (c) 6 and (d) 24 h. Black and white bars represent S-SPIONs and A-SPIONs, respectively.

using MTT assay. The results revealed that both S-SPIONs and A-SPIONs did not affect viability of MCF-7 cells; cell viability remained $>80 \%$ for all conditions. Increasing of FBS concentration to $10 \%$ slightly increased cell viability of A-SPIONs at low concentration of 0.05 and $0.1 \mathrm{mg} / \mathrm{ml}$. This could be due to the stabilizing effect of serum proteins to A-SPIONs according to the prevention of aggregates formation. In addition, A-SPIONs showed increase cell viability over $100 \%$ only when treated at high concentration of $0.4 \mathrm{mg} / \mathrm{ml}$ in all conditions. Hence it could be suggested that the surface coating of both S- and A-SPIONs could prevent aggregate formation and did not cause cytotoxicity to the cells.

\section{CONCLUSIONS}

In conclusion, aqueous-stabilized SPIONs were synthesized with silica and amine surface modification. Dispersion of the NPs in cell culture media altered their surface properties and resulted in aggregations. Supplementation of serum proteins enhanced NPs stability which could be due to the formation of protein coronas. Silica and amine surface coatings showed surface stabilization with no cytotoxicity towards MCF-7 cells. Hence these stable nontoxic S-SPIONs and A-SPIONs would be suitable for further conjugation with antibodies, peptides, or other biomolecules to improve specificity towards cancer cells which could be applied as contrasting agents in magnetic resonance imaging or as drug delivery system for cancer diagnosis or therapeutic applications.

Acknowledgements: We thank Assistant Professor Nuttawee Niamsiri, Department of Biotechnology, Faculty of Science, Mahidol University for DLS instrument support. This project was supported by Mahidol University, the Faculty of Science, Mahidol University and the Thailand Research Fund (IRG5980008, RTA5980001, MRG5980041). YT and WM were supported by a Science Achievement Scholarship of Thailand. This project was partially supported by a CIF grant, Center of Nanoimaging and DKSH Center of Excellence, Faculty of Science, Mahidol University.

\section{REFERENCES}

1. Guggenheim EJ, Khan A, Pike J, Chang L, Lynch I, Rappoport JZ (2016) Comparison of confocal and 
super-resolution reflectance imaging of metal oxide nanoparticles. PLoS One 11, e0159980.

2. Borlido L, Azevedo AM, Sousa AG, Oliveira PH, Roque AC, Aires-Barros MR (2012) Fishing human monoclonal antibodies from a CHO cell supernatant with boronic acid magnetic particles. $J$ Chromatogr $B$ 903, 163-70.

3. Xu H, Aguilar ZP, Yang L, Kuang M, Duan H, Xiong Y, Wei H, Wang A (2011) Antibody conjugated magnetic iron oxide nanoparticles for cancer cell separation in fresh whole blood. Biomaterials 32, 9758-65.

4. Javid A, Ahmadian S, Saboury AA, Kalantar SM, RezaeiâĂŘZarchi S (2013) Chitosan-coated superparamagnetic iron oxide nanoparticles for doxorubicin delivery: synthesis and anticancer effect against human ovarian cancer cells. Chem Biol Drug Des 82, 296-306.

5. Ansari L, Malaekeh-Nikouei B (2017) Magnetic silica nanocomposites for magnetic hyperthermia applications. Int J Hyperthermia 33, 354-63.

6. Quinto CA, Mohindra P, Tong S, Bao G (2015) Multifunctional superparamagnetic iron oxide nanoparticles for combined chemotherapy and hyperthermia cancer treatment. Nanoscale 7, 12728-36.

7. Na Y, Yang S, Lee S (2014) Evaluation of citratecoated magnetic nanoparticles as draw solute for forward osmosis. Desalination 347, 34-42.

8. Petcharoen K, Sirivat A (2012) Synthesis and characterization of magnetite nanoparticles via the chemical co-precipitation method. Mater Sci Eng B 177,
421-7.

9. Moore TL, Rodriguez-Lorenzo L, Hirsch V, Balog S, Urban D, Jud C, Rothen-Rutishauser B, Lattuada M, et al (2015) Nanoparticle colloidal stability in cell culture media and impact on cellular interactions. Chem Soc Rev 44, 6287-305.

10. Dhadge VL, Hussain A, Azevedo AM, Aires-Barros $\mathrm{R}$, Roque AC (2014) Boronic acid-modified magnetic materials for antibody purification. $J R$ Soc Interface 11, 20130875.

11. Yu S, Chow GM (2004) Carboxyl group $\left(-\mathrm{CO}_{2} \mathrm{H}\right)$ functionalized ferrimagnetic iron oxide nanoparticles for potential bio-applications. J Mater Chem 14, 2781-6.

12. Maneeprakorn W, Maurizi L, Siriket H, Wutikhun T, Dharakul T, Hofmann H (2017) Superparamagnetic nanohybrids with cross-linked polymers providing higher in vitro stability. J Mater Sci 52, 9249-61.

13. Prapainop K, Wentworth P (2011) A shotgun proteomic study of the protein corona associated with cholesterol and atheronal-B surface-modified quantum dots. Eur J Pharm Biopharm 77, 353-9.

14. Prapainop K, Witter DP, Wentworth P (2012) A chemical approach for cell-specific targeting of nanomaterials: small-molecule-initiated misfolding of nanoparticle corona proteins. J Am Chem Soc 134, 4100-3.

15. Jang JH, Lim HB (2010) Characterization and analytical application of surface modified magnetic nanoparticles. Microchem J 94, 148-58. 